\title{
THE SUN'S ROTATION NEAR THE INTERFACE BETWEEN ITS CONVECTIVE AND RADIATIVE ZONES: 1986 TO 1990
}

\author{
PHILIP R. GOODE \\ Department of Physics, New Jersey Institute of Technology, \\ Newark, New Jersey
}

The Sun's rotation rate near the base of its convection zone might be expected to vary over the solar cycle because of related changes there in the magnetic field. Helioseismic analyses have taught us that much of the Sun's convection zone rotates with surface-like differential rotation and a transition toward solid body rotation beneath. For a review of what we know about the Sun's internal rotation, see Goode, et al.(1991). We now have sufficient solar oscillation data to look for changes in the internal rotation near the base of the convection zone. The relevant data are from the 1986, 1988, 1989 and 1990 Big Bear Solar Observatory( BBSO) sets, Libbrecht and Woodard(1992, private communication). These four datasets were gathered at the same site for roughly the same number of days, reduced in the same way and span the same temporal and spatial frequency ranges--the differences between the sets should arise primarily because they were obtained in different years.

The splitting data for the five minute acoustic modes are defined by

$$
\nu_{n, l, m}-\nu_{n, l, 0}=L \sum_{i=1}^{N} a_{i, n, l} P_{i}\left(\frac{m}{L}\right),
$$

where $\nu_{n, l, m}$ is the frequency of the oscillation, $P_{i}$ is a Legendre polynomial, and the $a_{i, n, l} l s$ are the splitting coefficients and where $n, l$ and $m$ are the radial order, angular degree and angular order of the oscillation, respectively. We consider a rotation law of the form

$$
\Omega(r, \theta)=\Omega_{0}(r)+\Omega_{1}(r) \cos ^{2} \theta+\Omega_{2}(r) \cos ^{4} \theta,
$$

where $\theta$ is the co-latitude. Over the region sampled by a particular $(n l)$ multiplet oscillation, the mode weighted rotation law in the equatorial plane is well approximated by

$$
\frac{<\Omega_{0}>}{2 \pi}=a_{1}+a_{3}+a_{5} .
$$

Along the polar axis, the equivalent mean is given by

$$
\frac{\left.<\Omega_{0}>+<\Omega_{1}>+<\Omega_{2}\right\rangle}{2 \pi}=a_{1}-4 a_{3}+8 a_{5} .
$$


If the rotation rate were independent of latitude, then $a_{3}$ and $a_{5}$ would be equal to zero. In fact, they are non-zero and tend to have opposite signs. The calculated rotation in the convection zone varies smoothly between the extremes of (3) and (4). From this and the fact the rate in the outer radiative interior is solid body-like, we expect that the size and perhaps the sign of the gradient in rotation near the base of the convection zone depends on latitude.

We study the latitudinal behavior of this gradient by calculating the internal rotation of the Sun from the four years of BBSO data employing the least squares method of regularized inversion used by Goode, et al.(1991) in their Fig. 2. They allowed a discontinuity in the rotation rate at an arbitrary radius and found that the best minimization resulted when the discontinuity was at the base of the convection zone. Here we perform the same type of inversion with the discontinuity assumed to be at the base of the convection zone. In Table I, we show the decrease in rotation rate going inwards across the jump from the convection zone into the radiative interior. Noting that the jump is positive in the equatorial plane and negative along the polar axis, while varying smoothly in between, we also show the latitude at which the gradient vanishes.

Table 1. Outward Going Jump in Equatorial and Polar Rotation Rate $(n \mathrm{~Hz})$ by Year and the latitude at which there is no jump.

$\begin{array}{cccc}\text { Year } & \text { Equatorial Jump } & \text { Polar Jump } & \text { No Jump Latitude } \\ 1986 & 18.4 \pm 9.4 & -105.5 \pm 55.9 & 30.1^{\circ} \\ 1988 & 21.0 \pm 9.2 & -102.6 \pm 55.2 & 35.9^{\circ} \\ 1989 & 16.6 \pm 9.5 & -82.4 \pm 54.6 & 29.2^{\circ} \\ 1990 & 14.9 \pm 10.5 & -86.3 \pm 54.0 & 26.1^{\circ}\end{array}$

We see that there are significant, but opposite changes in rotation near the base of the convection zone at high and low latitudes. These changes are consistent with an abrupt transition from surface-like differential rotation in the convection zone to solid body-like rotation beneath. With some imagination, one sees a trend for the size of the jump to decrease with increasing time away from solar minimum. With even more imagination, considering the relatively larger uncertainties, one could see a similar trend in the latitude at which the gradient vanishes. This latitude is near the highest active latitudes. A further clue to the nature of the interface region comes from defining $L(r)$, following Dziembowski and Goode(1992),

$$
L(r)=\int_{0}^{1} \Omega(r, \theta)\left(1-\mu^{2}\right) d \mu, \quad \mu=\cos \theta,
$$

and $L$ is related to the total angular momentum $L_{T}$, by

$$
L_{T}=\int_{0}^{R} L(r) \rho r^{4} d r
$$


In the same limit that led to (3) and (4), we find

$$
<L(r)>=\frac{2}{3} a_{1}
$$

The $a_{1}^{\prime} s$ are fairly $l$-independent which means that $L(r)$ shows essentially no jump across the convective/radiative interface. Thus, the rotation rate as a function of latitude is re-arranged so there is no net torque across the interface. This result is consistent with the jump in rotation rate having opposite signs at high and low latitudes.

In Figure 1, we show the rotation rate as a function of radius for each of the four BBSO datasets. From these data, the rates are most reliable between 0.6 and $0.9 R_{0}$. The rates are shown at the latitude at which the rotation rate is continuous across the interface. We chose this latitude because the effect of the jump will be minimized in an inversion. We note that the four calculated laws look quite similar, except for an offset, with each showing a bump between 0.8 and $1.0 R_{0}$ while each also shows a gradual decline beneath the base of the convection zone $\left(0.72 R_{0}\right)$ to about $0.6 R_{0}$. There is relative structure between the base of the convection zone and about $0.8 R_{0}$. Thus, we speculate that the cycle dependent changes occur just above the base of the convection zone. This is consistent with the conclusion of Kosovichev and Gough(1992) for the equatorial plane. Below $0.5 R_{\mathrm{o}}$, it is difficult to know if the trend in the 1986 data is real at this latitude.

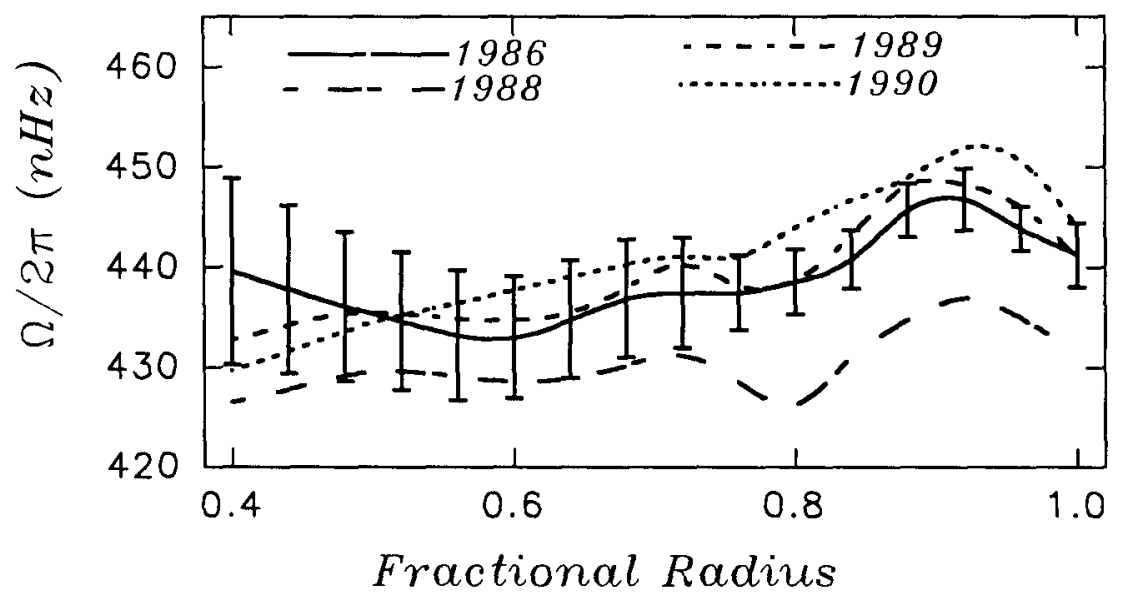

Fig. 1. The Sun's internal equatorial rotation laws $(n \mathrm{~Hz})$ at the latitude where there is no jump at the convective/radiative interface vs. fractional radius. The four laws are calculated from the 1986, 1988, 19891990 splitting data of Libbrecht and Woodard(1992). A jump in the rotation is allowed at the base of the convection zone. 


\section{Reference List}

Dziembowski, W.A. and Goode, P.R., 1992, GONG proceedings, in preparation.

Goode, P.R., Dziembowski, W.A., Korzennik, S.G. and Rhodes, E.J. 1991, Ap. J., , 367, 649 .

Gough, D.O. and Kosovichev, A.G., 1992, IAU Colloq. 137, in press.

Libbrecht, K.G. and Woodard, M.F., 1992, private communication. 